\title{
Nosocomial Providencia stuartii Meningitis: A Case Report
}

\author{
Ayhan Tekiner, Zeliha Kocak Tufan ${ }^{\mathrm{b}, \mathrm{c}}$, Mehmet Akif Bayar, Tuncer Tascioglu ${ }^{\mathrm{a}}$, Yavuz Selim \\ Erkoc $^{\mathrm{a}}$, Cigdem Hatipoglu ${ }^{\mathrm{b}}$
}

\begin{abstract}
Providencia stuartii is an opportunistic pathogen and may cause health-care infections. They mostly cause urinary-catheter-related infections. Meningitis associated with this bacterium is extremely rare. Here we report a $P$. stuartii meningitis in a patient with external lumbar drainage in the neurosurgery unit. A fifty-seven-year old male patient was admitted to the neurosurgery department with headache and confusion. There was a subarachnoidal hemorrhage on computerized tomography (CT) scan and he was transferred to the intensive care unit. His neurological evaluation showed a grade 3a mental status according to the Yasargil classification and a Glasgow coma scale of 14 . The CT-angiography and digital subtraction angiography revealed multiple arterial aneurisms. Coil embolization was made for three of the aneurisms. Since the patient had hydrocephalus on his follow-up on the 14th day, a lumbar drainage (LD) catheter was inserted. Daily cerebrospinal fluid (CSF) analyses were performed. On the 7th day of the LD the CSF findings revealed meningitis and $P$. stuartii was revealed from the three subsequent CSF cultures. The LD was removed and daily lumbar punctures and CSF cultures were performed. On the 7th day of his antibiotic therapy his laboratory findings returned to normal levels. Following CSF cultures were negative. The antibiotic therapy continued to 21 days. His meningitis was cured. To the authors' knowledge there were only two patients with $P$. stuartii meningitis in the literature and this is the third one.
\end{abstract}

Keywords: Providencia stuartii; Nosocomial meningitis; Emerging bacteria; External lumbar drainage

Manuscript accepted for publication March 16, 2011

${ }^{a}$ Neurosurgery Department, Ankara Training and Research Hospital, Ulucanlar, 06340 Ankara, Turkey

${ }^{\mathrm{b}}$ Infectious Diseases and Clinical Microbiology Department, Ankara Training and Research Hospital, Ulucanlar, 06340 Ankara, Turkey

${ }^{\mathrm{c} C}$ Corresponding author: Zeliha Kocak Tufan,

Email: drztufan@yahoo.com,drzeliha@windowslive.com

doi:10.4021/jnr105e

\section{Introduction}

Providencia is a Gram-negative bacterium and is usually found in soil and water. The genus comprises five species: P. stuartii, P. rettgeri, P. rustigianii, P. alcalifaciens and $P$. heimbachae [1]. Providencia infections are very rare and usually they cause health care associated infections. In recent years, although this species usually preserve their susceptibility to extended cephalosporins, extended spectrum beta lactamase (ESBL) production has emerged among these genera [2]. Especially P. stuartii and $P$. rettgeri have gained clinical importance because of their marked tendency to become resistant to antimicrobial agents and to cause nosocomial infections [3].

Nosocomial infections are common in intensive care units. Concerning the neurosurgery ICU, the incidence of urinary tract infections and catheter related infections may be similar to the other ICUs but other than those the risk of nosocomial meningitis also exists in neurosurgery ICU [4].

$P$. stuartii is mostly found to cause urinary catheter related infections. Meningitis associated with this bacterium is extremely rare. Here we report a $P$. stuartii meningitis in a patient with external lumbar drainage in a neurosurgery unit.

\section{Case Report}

A fifty-seven-year-old male patient was admitted to our neurosurgery department with headache and confusion. Subarachnoidal hemorrhage was found on computerized tomography (CT) scan and he was transferred to the intensive care unit. His neurological evaluation showed a grade 3 a mental status according to the Yasargil classification and a Glasgow coma scale (GCS) of 14. Nimodipine, phenytoin, dexamethasone, famotidine and diazepam therapies were administered. The CT angio and digital subtraction angiography (DSA) revealed multiple arterial aneurisms. Coil embolization was performed for three of the aneurisms. Since the patient had hydrocephalus on his follow-up on the 14th day, a lumbar drainage (LD) catheter was inserted. Daily cerebrospinal fluid (CSF) analyses and cultures were performed. On the 21st 
day of his admission and 7th day of the LD, he developed fe$\operatorname{ver}\left(38.1^{\circ} \mathrm{C}\right)$. He was in stupor; nuchal rigidity was negative; Kernig and Brudzinski signs could not evaluated. His white blood cell count increased to $12.5 \times 10^{3} / \mu \mathrm{L}$ from $7.5 \times 10^{3} /$ $\mu \mathrm{L}$. The hemoglobin, hematocrit and platelet counts were as follows: $10.8 \mathrm{~g} / \mathrm{dL}, 31 \%, 41 \times 10^{3} / \mu \mathrm{L}$. The CSF examination revealed meningitis findings: 400 leucocyte $/ \mathrm{mm}^{3}(90 \%$ polymorphonuclear leucocytes), glucose: $31 \mathrm{mg} / \mathrm{dL}$ (serum glucose level was $166 \mathrm{mg} / \mathrm{dL}), \mathrm{Cl}: 120 \mathrm{mmol} / \mathrm{L}$, protein: 2,365 $\mathrm{mg} / \mathrm{L}$ (normal range: 150 - $450 \mathrm{mg} / \mathrm{L}$ ). Gram staining of the CSF revealed Gram-negative bacilli. Ceftriaxone $2 \times 2$ gr was started. CSF cultures were sent ever day. Providencia stuartii was revealed from three subsequent CSF cultures. The organism was resistant to gentamycine, tobramycine, ciprofloxacine, levofloxacine, colistin and ampisillin-sulbactam. It was susceptible to ceftriaxone, carbapenems, trimethoprim-sulfamethoxazole and piperacillin-tazobactam. The drainage catheter was removed and daily lumbar punctures were performed instead. His blood and urinary cultures were negative. His antibiotic therapy was completed to 21 days and the laboratory findings of the blood and CSF returned to normal levels. Because of the neurosurgical care he stayed in the hospital one month more and then he was discharged.

\section{Discussion}

We describe a patient with nosocomial meningitis associated with $P$. stuartii. $P$. stuartii is a very rare pathogen in community acquired infections and usually isolated from the urine in nosocomial urinary tract infections. In one study the overall hospital incidence was found to be 0.008 per 1000 hospital admissions/year and it was isolated in 223 of 262,364 patients from the following specimens: urine (87\%), blood $(10 \%)$ and respiratory tract secretions (3\%) [2]. In the same study the ESBL production was found to be $52 \%$. Advanced age, previous hospitalization and previous antibiotic use were the risk factors for ESBL producing Providencia. According to the infection control committee reports regarding ICUs of our hospital, there was only one patient with Providencia infection which was a urinary catheter related infection in 2009. Our meningitis patient was the only patient with Providencia infection in the ICUs since then.

As mentioned before the main infection associated with Providencia is UTIs followed by bacteremia and pneumonia. Besides this a pericarditis and an endocarditis case were reported $[5,6]$. To the authors' knowledge there were only two patients with $P$. stuartii meningitis in the literature up to now. Scapellato et al reported a 46-year-old man who presented with subarachnoid hemorrhage and intraventricular bleeding. After the patient underwent a craniotomy operation he developed Enterococcus faecium meningitis and after a while also $P$. stuartii meningitis. The patient was treated with imipenem for P. stuartii meningitis [7]. The second case was reported very recently from Turkey. The patient had an external lumbar drain because of a CSF leakage and on the 40th day of admission he developed P. stuartii meningitis [7, 8]. The patient was treated with meropenem.

Providencia is usually found in soil and water. When the bacteria caused an outbreak in a burn unit, the clonality of the bacteria was also shown from different components of the hospital instruments like the aspirator (probe, reservoir and tube) [9]. In our case we did not found the source but when the patient was in the ICU, neurosurgery ICU was under construction and the patient had been transferred to another ICU and then turned back to the neurosurgery ICU. The exact source remained unknown. In 2010 we did not have any other Providencia infection in any of other ICUs following this patient.

The emerging antibacterial resistance is a very important situation in $P$. stuartii. In one study concerning the ESBL production of different enterobacteriacea species, $P$. stuartii had as high as $18.8 \%$ ESBL production [10] and 52\% in another study [2]. ESBL PER1 positive $P$ stuartii was identified from Italy, Kosova and Algeria. VIM 1 metallo beta lactamase, CTX M, TEM 52, and TEM 72 type beta lactamase were other resistance mechanisms shown in P. stuartii [2, $10-12]$. In our case the strain was susceptible to many antibiotics including ceftriaxone. So we continued the empirical therapy which was ceftriaxone and the patient was cured.

In conclusion, we suggest that the emerging of this bacterium in hospital settings and the potential resistance of the bacteria have to be kept in mind and it has to be considered in nosocomial meningitis cases.

\section{Conflict of Interest}

None

\section{References}

1. Donnenberg MS. Enterobacteriaceae. In: Mandell GL, Bennett JE, Dolin R, editors. Mandell, Douglas, and Bennett's principles and practice of infectious diseases. Philadelphia: Churchill Livingstone. 2010; pp. 28152833.

2. Tumbarello M, Citton R, Spanu T, Sanguinetti M, Romano L, Fadda G, Cauda R. ESBL-producing multidrugresistant Providencia stuartii infections in a university hospital. J Antimicrob Chemother 2004;53(2):277-282.

3. Fischer R, Penner JL, Zurinaga G, Riddle C, Samisch W, Brenner DJ. Usefulness of trehalose fermentation and L-glutamic acid decarboxylation for identification of biochemically aberrant Providencia stuartii strains. J Clin Microbiol 1989;27(9):1969-1972.

4. Arunodaya GR. Infections in neurology and neurosur- 
gery intensive care units. Neurol India 2001;49 Suppl 1:S51-59.

5. Simon C, Dieli M, Brucato A, Pedrotti P, Brambilla P, Curri SF, Senni M, et al. Images in cardiovascular medicine. Bacterial pericarditis due to Providencia stuartii: an atypical case of relapsing pericarditis. Circulation 2010;122(4):e401-403.

6. Krake PR, Tandon N. Infective endocarditis due to Providenca stuartii. South Med J 2004;97(10):1022-1023.

7. Scapellato PG, Ormazabal C, Scapellato JL, Bottaro EG. Meningitis due to vancomycin-resistant Enterococcus faecium successfully treated with combined intravenous and intraventricular chloramphenicol. J Clin Microbiol 2005;43(7):3578-3579.

8. Sipahi OR, Bardak-Ozcem S, Ozgiray E, Aydemir S, Yurtseven T, Yamazhan T, Tasbakan M, et al. Meningitis due to Providencia stuartii. J Clin Microbiol
2010;48(12):4667-4668.

9. Saida NB, Thabet L, Messadi A, Bouselmi K, Turki A, Boukadida J. Clonality of Providencia stuartii isolates involved in outbreak that occurred in a burn unit. Burns 2008;34(6):829-834.

10. Kader AA, Kamath KA, Dass SM. Accelerated detection of extended-spectrum beta-lactamases in clinical isolates of Enterobacteriaceae. Br J Biomed Sci 2006;63(4):151154.

11. Miriagou V, Tzouvelekis LS, Flevari K, Tsakiri M, Douzinas EE. Providencia stuartii with VIM-1 metallo-betalactamase. J Antimicrob Chemother 2007;60(1):183184.

12. Minarini LA, Gales AC, Palazzo IC, Darini AL. Prevalence of community-occurring extended spectrum betalactamase-producing Enterobacteriaceae in Brazil. Curr Microbiol 2007;54(5):335-341. 